TPI-MINN-00/53-T

UMN-TH-1929/00

FTUV/001113

IFIC/00-70

\title{
Domain walls in supersymmetric QCD: The taming of the zoo
}

\author{
Daniele Binosi \\ Departamento de Física Teórica and IFIC, Centro Mixto, \\ Universidad de Valencia-CSIC, \\ E-46100, Burjassot, Valencia, Spain
}

Tonnis ter Veldhuis

Theoretical Physics Institute, Univ. of Minnesota, Minneapolis, MN 55455

\begin{abstract}
We provide a unified picture of the domain wall spectrum in supersymmetric QCD with $N_{c}$ colors and $N_{f}$ flavors of quarks in the (anti-) fundamental representation. Within the framework of the Veneziano-Yankielowicz-Taylor effective Lagrangian, we consider domain walls connecting chiral symmetry breaking vacua, and we take the quark masses to be degenerate. For $N_{f} / N_{c}<1 / 2$, there is one BPS saturated domain wall for any value of the quark mass $m$. For $1 / 2 \leq N_{f} / N_{c}<1$ there are two critical masses, $m_{*}$ and $m_{* *}$, which depend on the number of colors and flavors only through the ratio $N_{f} / N_{c}$; if $m<m_{*}$, there are two BPS walls, if $m_{*}<m<m_{* *}$ there is one non-BPS wall, and if $m>m_{* *}$ there is no domain wall. We numerically determine $m_{*}$ and $m_{* *}$ as a function of $N_{f} / N_{c}$, and we find that $m_{* *}$ approaches a constant value in the limit that this ratio goes to one.
\end{abstract}

E-mail: binosi@hal.ific.uv.es; veldhuis@hep.umn.edu 


\section{Introduction}

In recent years, several groups have studied domain walls in supersymmetric quantum chromodynamics (SQCD) in the framework of the Veneziano-Yankielowicz-Taylor (VYT) effective Lagrangian [1]. This Lagrangian is not an effective Lagrangian in the usual Wilsonian sense, but it it is expected to describe the vacuum structure of SQCD. If the masses of the quark matter fields are degenerate, the VYT effective Lagrangian takes the form of a Wess-Zumino model in terms of the chiral superfields $\Phi$, representing the glueball superfield, and $X$, representing the quark matter. The effective Lagrangian has $N_{c}$ chiral symmetry breaking vacua and an additional chiral symmetry conserving vacuum. The spectrum of the domain walls connecting two adjacent chiral symmetry breaking vacua was found to depend on the number of colors $N_{c}$, the number of flavors $N_{f}$, and the common mass of the quarks $m$. In Ref.[2], domain walls were studied in several models with $N_{f}=N_{c}-1$. In the cases of $N_{c}=2,3$ and 4 , it was found that for small quark mass $m$, there are two Bogomolnyi-Prasad-Sommerfield (BPS) saturated domain walls interpolating between two adjacent vacua. Above a first critical mass $m_{*}$, these BPS walls combine and form one non-BPS wall. If the mass is higher than a second critical value $m_{* *}$, there is no domain wall left; the two adjacent vacua can only be connected by a two wall configuration passing through the chiral symmetry conserving vacuum. In Ref.[3] it was found that for any theory with $N_{f} / N_{c}<1 / 2$ there is one BPS wall connecting adjacent chiral symmetry breaking vacua both in the small $m$ limit and in the large $m$ limit.

The purpose of this work is to provide a unified description of the domain wall spectrum of the VYT effective Lagrangian for any value of $N_{c}$ and $N_{f}$. One key observation we make is that, with a canonical Kähler potential, the static solutions to the equations of motion take the form

$$
\begin{aligned}
\phi & =\phi\left(N_{c} z, m, N_{f} / N_{c}\right), \\
\chi & =\chi\left(N_{c} z, m, N_{f} / N_{c}\right) .
\end{aligned}
$$

Here $\phi$ and $\chi$ are the scalar components of the superfields $\Phi$ and $X$ respectively. Theories with different values of $N_{c}$ but identical ratios of $N_{f} / N_{c}$ have domain wall solutions that differ only by a scale factor $N_{c}$. Therefore, only theories with mutually prime values of $N_{c}$ and $N_{f}$ need to be considered. Theories for which $N_{c}$ and $N_{f}$ have common divisors are related to theories with lower values of $N_{c}$ and $N_{f}$.

We also make use of the fact that BPS states lie in shortened multiplets of the centrally extended supersymmetry algebra. Only two shortened multiplets can combine to form one regular multiplet, and therefore the number of BPS saturated domain walls can only change by a multiple of two when the mass parameter is continuously varied [ 4 ]. For small values of $m$, we show that if $N_{f} / N_{c}<1 / 2$ there is one BPS wall, and if $1 / 2 \leq N_{f} / N_{c}<1$ there are two BPS walls. It then follows that the number of BPS walls is odd for any value of $m$ if $N_{f} / N_{c}<1 / 2$, and even for any value of $m$ if $1 / 2 \leq N_{f} / N_{c}<1$. Note that this is fully consistent with the previous results in Refs.[2], 3]. 
Even though the single BPS wall at small $m$ for $N_{f} / N_{c}<1 / 2$ can not disappear when the mass is increased, one can not exclude the possibility of the appearance and subsequent disappearance of a new pair of BPS walls at intermediate values of $m$. We therefore perform a numerical study of this class of theories, and find that such an exotic event does not occur.

For theories with $1 / 2 \leq N_{f} / N_{c}<1$, the fact that the number of BPS wall is even does not block the disappearance of the pair of degenerate BPS walls that exist at small $m$. Indeed, in the specific cases previously studied [2] this happens at the mass $m_{*}$. We also perform a numerical study of the theories in this class, and find that the prototype behavior found in Ref.[2] is valid in general. These theories all have a critical mass $m_{*}$ at which the two BPS walls combine to form a non-BPS wall, and a second critical mass $m_{* *}$, where the non-BPS wall disappears. It is clear from the general form of the static solutions in Eq.(11) that the values of $m_{*}$ and $m_{* *}$ only depend on $N_{f}$ and $N_{c}$ through the ratio $N_{f} / N_{c}$.

The remainder of this paper is organized as follows. In Section 1 we introduce the VYT Lagrangian as an effective description of SQCD. Next, in Section 2 we discuss domain walls in the VYT model, followed by the presentation of our numerical results in Section 3 and 4. We first show that in the theories with $N_{f} / N_{c}<1 / 2$ there is one BPS wall for all values of $m$. Then, for the theories with $1 / 2 \leq N_{f} / N_{c}<1$, we determine $m_{*}$ and $m_{* *}$ as a function of $N_{f} / N_{c}$. In the final section we discuss how our results depend on some of the assumptions we made, and to what extend the results we derived in the context of the VYT effective Lagrangian apply to SQCD.

\section{Supersymmetric QCD and the VYT effective Lagran- gian}

The fundamental Lagrangian describing SQCD, extensively discussed in for example the review [5], takes the form

$$
\begin{aligned}
\mathcal{L}= & \left(\frac{1}{4 g^{2}} \operatorname{tr} \int d^{2} \theta W^{2}+\text { h.c. }\right)+\left(\frac{1}{4} \int d^{4} \theta Q^{i \dagger} \mathrm{e}^{V} Q_{i}+\frac{1}{4} \int d^{4} \theta \bar{Q}^{j \dagger} \mathrm{e}^{-V} \bar{Q}_{j}\right) \\
& -\left(\frac{m^{i j}}{2} \int d^{2} \theta \bar{Q}_{i} Q_{j}+\text { h.c. }\right) .
\end{aligned}
$$

Here color indices have been suppressed, and $i=1, \ldots, N_{f}$ is a flavor index. The vector superfield $V$ contains the gauge bosons and gauginos, and $Q_{i}$ and $\bar{Q}_{i}$ are the quark chiral superfields transforming respectively under the $N_{c}$ and $\bar{N}_{c}$ representations of the $S U\left(N_{c}\right)$ gauge group. In addition, $g$ is the gauge coupling, and $m^{i j}$ is the quark mass matrix. If $N_{f} \leq N_{c}-1$ then the theory has a dynamically generated superpotential. In the absence of a quark mass, the model has a $S U\left(N_{f}\right) \otimes S U\left(N_{f}\right) \otimes U(1)_{V} \otimes U(1)_{A} \otimes U(1)_{R}$ global symmetry at the classical level. The $U(1)_{R}$ symmetry is broken down to the discrete symmetry $\mathbb{Z}_{2 N}$ by an anomaly. It is further broken down spontaneously to $\mathbb{Z}_{2}$ when a gaugino condensate $(\operatorname{tr} \lambda \lambda)$ is formed. 
The VYT effective Lagrangian proposed in Ref.[1] and modified in Ref.[6] to properly realize the discrete anomaly free $\mathbb{Z}_{2 N}$ symmetry, provides a partial description of SQCD with $N_{f}<N_{c}$ at low energy in terms of the composite chiral superfields

$$
\Phi^{3}=\frac{3}{32 \pi^{2}} \operatorname{tr} W^{2}, \quad M_{i j}=2 \bar{Q}_{i} Q_{j},
$$

representing the glueball and matter chiral superfields, respectively.

The superpotential of the model, which is constructed to reproduce the anomalous Ward identities of SQCD, takes the form

$$
\mathcal{W}=\frac{2}{3} \Phi^{3}\left[\log \left(\frac{\Phi^{3\left(N_{c}-N_{f}\right)} \operatorname{det} M}{\Lambda^{3 N_{c}-N_{f}}}\right)-\left(N_{c}-N_{f}\right)\right]-\frac{1}{2} \operatorname{tr}(m M) .
$$

The VYT effective Lagrangian is not an effective theory in the usual Wilsonian sense. It does not describe all relevant low energy degrees of freedom, but it reproduces Green's functions involving only the $\Phi$ and the $M$ fields [6].

We take the quark mass matrix to be diagonal and degenerate, $m^{i j}=m \delta^{i j}$. We then assume that for the purpose of studying the vacuum structure and domain walls, we can take $M_{i j}=X^{2} \delta_{i j}$. In terms of $\mathrm{X}$, the superpotential reads

$$
\mathcal{W}=\frac{2}{3} \Phi^{3}\left[\log \left(\frac{\Phi^{3\left(N_{c}-N_{f}\right)} X^{2 N_{f}}}{\Lambda^{3 N_{c}-N_{f}}}\right)-\left(N_{c}-N_{f}\right)\right]-\frac{m}{2} N_{f} X^{2} .
$$

The Kähler potential is not uniquely determined by symmetry considerations. For now, we take it to have the canonical form

$$
\mathcal{K}=\bar{\Phi} \Phi+\bar{X} X
$$

In Section 5 we will comment on how our results may change with a different form of the Kähler potential. The kinetic terms for the scalar components are

$$
\mathcal{L}_{\text {kin }}=\partial^{\mu} \phi \partial_{\mu} \phi^{*}+\partial^{\mu} \chi \partial_{\mu} \chi^{*}
$$

and the scalar potential is $(\Lambda=1$ from here on $)$

$$
V(\phi, \chi)=4\left|\phi^{2} \log \left[\phi^{3\left(N_{c}-N_{f}\right)} \chi^{2 N_{f}}\right]\right|^{2}+N_{f}^{2}\left|m \chi-\frac{4 \phi^{3}}{3 \chi}\right|^{2} .
$$

This potential has $N_{c}$ chiral symmetry breaking vacua, corresponding to the physical expectation values

$$
\begin{aligned}
\left\langle\phi^{3}\right\rangle_{l} & =\left(\frac{3 m}{4}\right)^{N_{f} / N_{c}} \mathrm{e}^{2 \pi i l / N_{c}}, \\
\left\langle\chi^{2}\right\rangle_{l} & =\left(\frac{4}{3 m}\right)^{\left(N_{c}-N_{f}\right) / N_{c}} \mathrm{e}^{2 \pi i l / N_{c}}
\end{aligned}
$$


where $l=0, . ., N_{c}-1$. In this paper we consider domain walls that interpolate between such vacua. In order to ensure that the two connected vacua lie on the same sheet of the logarithm in the scalar potential (8), we write the vacuum expectation values of $\phi$ and $\chi$ as

$$
\begin{aligned}
\langle\phi\rangle_{k}=\langle\phi\rangle_{0} \mathrm{e}^{-2 \pi i k N_{f} / 3 N_{c}}, & \langle\phi\rangle_{0} \equiv\left(\frac{3 m}{4}\right)^{N_{f} / 3 N_{c}}, \\
\langle\chi\rangle_{k}=\langle\chi\rangle_{0} \mathrm{e}^{\pi i k\left(N_{c}-N_{f}\right) / N_{c}}, & \langle\chi\rangle_{0} \equiv\left(\frac{4}{3 m}\right)^{\left(N_{c}-N_{f}\right) / 2 N_{c}},
\end{aligned}
$$

with $k=0, . ., N_{c}-1$. This ensures that the phase of the argument of the logarithm is equal to zero in the vacuum. Note that not all vacua can be written in the form of Eq.(10). For example, the vacuum with $l=1$ in Eq.(9) in the case $N_{c}=4$ and $N_{f}=2$ does not lie on the same sheet as the vacuum with $l=0$. The latter vacuum is obtained both for $k=0$ or 2 in Eq.(10). These two vacua can therefore not be connected by a domain wall, unless it crosses the branch cut. The SQCD gaugino condensates in the vacuum labeled by $k$ is

$$
\langle\operatorname{tr} \lambda \lambda\rangle_{k}=\frac{32 \pi^{2}}{3}\left\langle\phi^{3}\right\rangle_{k}=\frac{32 \pi^{2}}{3}\left(\frac{3 m}{4}\right)^{N_{f} / N_{c}} \mathrm{e}^{-2 \pi i k N_{f} / N_{c}} .
$$

Apart from the chiral symmetry breaking vacua, the VYT model also has a symmetric vacuum with $\phi=0$ and $\chi=0$, corresponding to vanishing gaugino condensate [6]. The existence of a corresponding vacuum in SQCD is very controversial.

In terms of the physical field $S=\Phi^{3}$, it is clear that the chirally symmetric vacuum in the VYT Lagrangian arises from a singularity in the metric that is derived from the Kähler potential (6), whereas the chiral symmetry breaking vacua are associated with extrema of the superpotential (5). As noted before, the Kähler potential is not completely determined by symmetry considerations, in contrast to the superpotential. Results that depend on the particular details of the Kähler potential are therefore suspect.

In favor of the chirally symmetric vacuum, it was argued in Ref.[6] that the existence of such a vacuum in combination with a vacuum averaging hypothesis could resolve a long standing inconsistency concerning gluino condensates calculated using the strong coupling instanton approach on the one side, and the weak coupling instanton approach on the other. However, in Ref.[7] it was shown that a similar inconsistency persists in supersymmetric QCD with light matter in the adjoint representation, even with the vacuum averaging hypothesis. As this theory is equivalent to softly broken $\mathcal{N}=2$ supersymmetric Yang-Mills theory (SYM), there is no uncertainty about its vacua if the mass of the adjoint is small. The result therefore casts doubt on the motivation for the chiral symmetry breaking vacuum in supersymmetric QCD.

In Ref.[8] it was stated that in the chiral symmetry conserving vacuum of the VYT Lagrangian certain discrete anomaly matching condition can not be satisfied. In Ref.[9] on the contrary it was argued that such considerations do not constrain the existence of a symmetric vacuum. 
Another piece of evidence pointing against the existence of a chirally symmetric vacuum in SQCD stems from deformed $\mathcal{N}=2$ supersymmetric theories. $\mathcal{N}=2 \mathrm{SYM}$ theory with a small mass for the adjoint has only two vacua. $\mathcal{N}=1 \mathrm{SYM}$ is obtained from this theory in the limit that the soft breaking mass goes to infinity. In Ref.[10, 11] it was thus argued that $\mathcal{N}=1 \mathrm{SYM}$ also has only two vacua, barring a phase transition. This argument indicates that there is no room for a third, chirally symmetric vacuum. A similar argument concerning SQCD with one flavor can be made by deforming $\mathcal{N}=2$ supersymmetric QCD with one hyper multiplet [12].

We conclude that it is dangerous to extend results obtained from the VYT effective Lagrangian to SQCD if these results depend on the existence of the chirally symmetric vacuum. In this article we only consider domain walls interpolating between chiral symmetry breaking vacua.

\section{Domain walls in the VYT effective Lagrangian}

Domain walls are static field configurations that interpolate between two vacua. They are static solutions to the Euler-Lagrange equations

$$
\frac{\partial^{2} \phi}{\partial t^{2}}-\frac{\partial^{2} \phi}{\partial z^{2}}=-\frac{\partial V}{\partial \phi^{*}}, \quad \frac{\partial^{2} \chi}{\partial t^{2}}-\frac{\partial^{2} \chi}{\partial z^{2}}=-\frac{\partial V}{\partial \chi^{*}}
$$

It can be checked that static solutions to these equations take the form of Eq.(1). We will refer to the theory with $N_{c}$ colors and $N_{f}$ flavors as $\left(N_{c}, N_{f}\right)$. It is clear that theories with the same ratio of $N_{f} / N_{c}$ have domain walls that only differ by a scale factor.

BPS saturated domain walls [13] in the VYT model satisfy the first order equations

$$
\frac{d \phi}{d z}=\mathrm{e}^{i \delta} \frac{\partial \overline{\mathcal{W}}}{\partial \phi^{*}}, \quad \frac{d \chi}{d z}=\mathrm{e}^{i \delta} \frac{\partial \overline{\mathcal{W}}}{\partial \chi^{*}} .
$$

Solutions to the BPS equation have an "integral of motion"

$$
I=\operatorname{Im}\left\{\mathrm{e}^{-i \delta} \mathcal{W}\right\}
$$

which does not depend on the space coordinate $z$.

We consider domain walls interpolating between adjacent vacua. For definiteness we take the vacua for $k=0$ and $k=1$. Note that these vacua are only neighboring in terms of the phase of the gaugino condensate if $N_{f}=1$ or $N_{f}=N_{c}-1$. Evaluation of the constant of motion $I$ in these two vacua provides the value of the phase $\delta$ in the BPS equation,

$$
\delta= \pm \frac{\pi}{2}-\pi \frac{N_{f}}{N_{c}}
$$

where the choice of the sign determines which of the two vacua is located at $z=+\infty$, and which at $z=-\infty$. The BPS bound on the energy of the interpolating domain wall is given by

$$
E_{\mathrm{BPS}}=2 N_{c}\left(\frac{4}{3}\right)^{1-N_{f} / N_{c}} m^{N_{f} / N_{c}} \sin \left(\pi \frac{N_{f}}{N_{c}}\right) .
$$




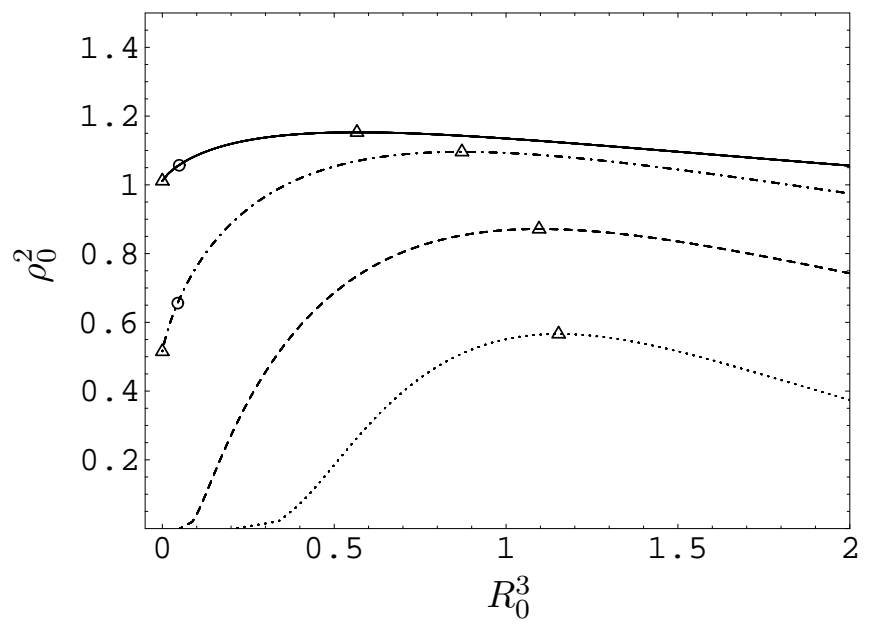

Figure 1: The solution to Eq.(19) for the $(5,1)$ (dotted), $(5,2)$ (dashed), (5,3) (dotteddashed) and $(5,4)$ (continuous) theory. Open triangles indicate the $m=0$ domain wall solution(s), while open circles represent the domain wall solution corresponding to $m=m_{*}$.

We parametrize the domain wall profiles in terms of four real functions by means of

$$
\begin{aligned}
& \phi(z)=\langle\phi\rangle_{0} R(z) \mathrm{e}^{i \beta(z)}, \\
& \chi(z)=\langle\chi\rangle_{0} \rho(z) \mathrm{e}^{i \alpha(z)} .
\end{aligned}
$$

If the domain wall profiles have reflection symmetry with respect to their center, then the integral of motion (14) evaluated at the center $(z=0)$ yields the following relation between the values of $R_{0} \equiv R(0)$ and $\rho_{0} \equiv \rho(0)$

$$
-R_{0}^{3}\left[\left(1-\frac{N_{f}}{N_{c}}\right)\left(\ln R_{0}^{3}-1\right)+\frac{N_{f}}{N_{c}} \ln \rho_{0}^{2}\right]-\frac{N_{f}}{N_{c}} \rho_{0}^{2}=\cos \left(\pi \frac{N_{f}}{N_{c}}\right) .
$$

Note that $N_{c}$ and $N_{f}$ enter in Eq.(19) only through the ratio $N_{f} / N_{c}$, and there is no dependence on $m$. A BPS saturated domain wall in the theory $\left(N_{c}, N_{f}\right)$ correspond to a point on a curve defined by Eq.(19) in the $\rho_{0}^{2}$ versus $R_{0}^{3}$ plane. There is one such curve for every value of $N_{f} / N_{c}$. In order to determine exactly where on the curve the point is located, the BPS equation needs to be solved explicitly. When the mass $m$ is varied and the domain wall remains BPS saturated, the point representing the wall will move along its curve.

We now consider the small mass limit $m \ll 1$, as previously discussed in Refs.[14, 3]. In this limit the field $\Phi$ can be integrated out by imposing the condition $\partial \mathcal{W} / \partial \phi=0$. In the resulting theory, which only contains the field $X$, there is one BPS wall interpolating between the $k=0$ and $k=1$ vacua. This wall corresponds to the maximum of the curve $\rho_{0}^{2}$ versus $R_{0}^{3}$ defined by Eq.(19). However, for certain values of $N_{c}$ and $N_{f}$ there is a

\footnotetext{
${ }^{1}$ It is amusing to observe that the maxima of the curves of the $\left(N_{c}, N_{f}\right)$ and $\left(N_{c}, N_{c}-N_{f}\right)$ theories are related by reflection in the line $\rho_{0}^{2}=R_{0}^{3}$.
} 
second possible BPS wall in the small mass limit for which $\phi \equiv 0$. This wall corresponds to the point

$$
R_{0}^{3}=0, \quad \rho_{0}^{2}=-\frac{N_{c}}{N_{f}} \cos \left(\pi \frac{N_{f}}{N_{c}}\right) .
$$

As $\rho_{0}$ is a modulus and therefore larger than or equal to zero, the second BPS wall is only present when $N_{f} / N_{c} \geq 1 / 2$.

We thus see that for small $m$ there is one BPS saturated wall if $N_{f} / N_{c}<1 / 2$, and there are two BPS walls if $1 / 2 \leq N_{f} / N_{c}<1$. Therefore, if $N_{f} / N_{c}<1 / 2$ the number of BPS walls must be odd for any value of the mass parameter $m$. In particular, the wall at small $m$ can not just disappear. Similarly, if $1 / 2 \leq N_{f} / N_{c}<1$, the number of BPS walls must be even. This allows the possibility of the simultaneous disappearance of the pair of BPS domain walls that exists at small $m$.

We illustrate this analysis in Fig. 11 for the theories with $N_{c}=5$ and $N_{f}=1,2,3$ and 4. We draw the four curves given by Eq.(19) in the $\rho_{0}^{2}$ versus $R_{0}^{3}$ plane, and indicate the location of the BPS walls at small $m$ on the curves with a triangle. For $N_{f}=1$ and 2 , there is only one triangle on the curve. When the mass $m$ is increased, these triangles start to move along the curve. How they move is a dynamical question which can only be answered by explicitly solving the BPS equations. It is clear though that they have to remain on their curve, they have nowhere else to go. In principle, there is no a priori argument that precludes the appearance of a new pair of BPS walls. As we will show in Section 3 , this does not occur. For $N_{f}=3$ and 4 there are two triangles on the curves. As we will show in Section 4 , when $m$ is increased, the two triangles start to move towards each other, and at the critical value $m_{*}$ they meet at the point on the curve indicated by a circle. They then combine to form a non-BPS wall.

\section{The case $N_{f} / N_{c}<1 / 2$}

The existence of one BPS wall at small $m$ for theories with $N_{f} / N_{c}<1 / 2$, in combination with the fact that the number of BPS walls modulo two is conserved, implies that the number of BPS walls in this class of theories is odd for any value of $m$. In order to determine whether any new pairs of BPS walls or additional non-BPS walls appear when $m$ is increased from zero, we performed a numerical simulation of all the theories in this class with $N_{c} \leq 8$.

We simulated the second order equations of motion on a lattice with a forward predicting algorithm. To that purpose, we transformed the Euler-Lagrange differential Eqs.(12) to finite difference equations, with discretized space and time coordinates. The scalar field $\phi$ and $\chi$ were kept fixed at their vacuum expectation values at the two ends of the lattice. The size of the lattice is chosen to be much larger than the width of the walls and, at the same time, the lattice spacing is chosen much smaller than the width. An initial configuration for $\phi$ and $\chi$ was constructed so that it interpolates between the two vacuum expectation values. This initial configuration does not satisfy the static equations of motion and therefore evolves in time. We are interested in a minimum energy con- 

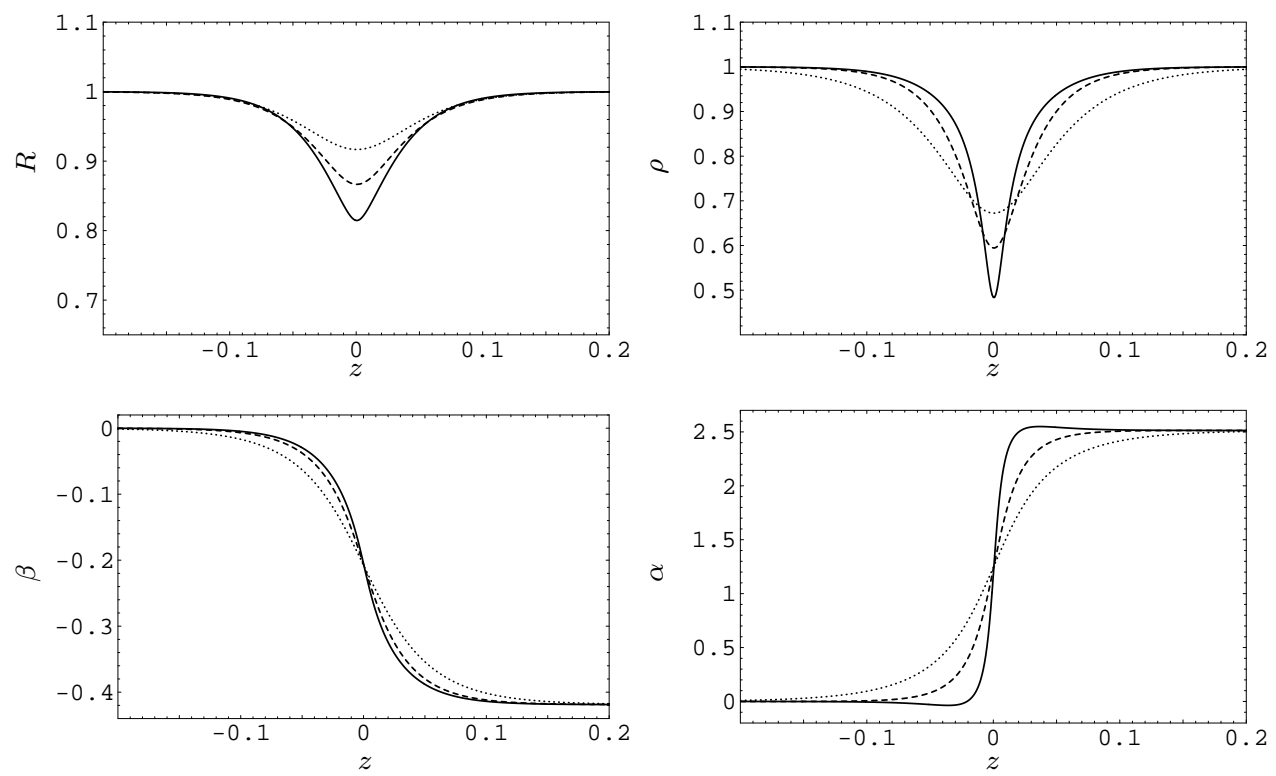

Figure 2: BPS domain walls for the $(5,1)$ theory. Masses are $m=10$ (dotted), 20 (dashed) and 50 (solid)

figuration. The system can only reach such a minimum energy configuration from the initial configuration if it can dissipate energy. We therefore added a friction term to the equations of motion. The coefficient of friction is adjusted so that the system relaxes to the minimum energy configuration in as short a time as possible. If the coefficient is too large, the system does not converge, and if it is too small, the systems keeps oscillating. Of course, as the friction terms are proportional to the time derivatives of the fields, the static configurations of the equations of motion with and without the friction term are the same. Once the system had come to rest, we calculated the energy of the final configuration and compared this to the BPS bound. We also explicitly checked that domain wall solutions we thus obtained did not cross the branch cut of the logarithm appearing in the scalar potential (8). This check was performed by plotting the argument of the logarithm, $\phi^{3\left(N_{c}-N_{f}\right)} \chi^{2 N_{f}}$, in the complex plane. As the fields on the domain wall vary from one vacuum to the other, this quantity traces a loop in the complex plane. We then verified that this loop does not cross the negative real axis (where the branch cut is located within our conventions).

We repeated this procedure for each theory, starting at low values of $m$ and following the walls as $m$ is increased. In order to see if more than one wall exists, we also repeated the procedure with various initial conditions. We found that for the theories in the class $N_{f} / N_{c}<1 / 2$ there is only one BPS saturated domain wall for any value of $m$. In Fig. 2 we show some representative examples of BPS domain walls for the $(5,1)$ theory and various values of $m$. Following the domain wall in this theory in Fig. 1, it starts on top of its curve at $m=0$, and then moves along the curve towards smaller values of $R_{0}^{3}$ when $m$ 


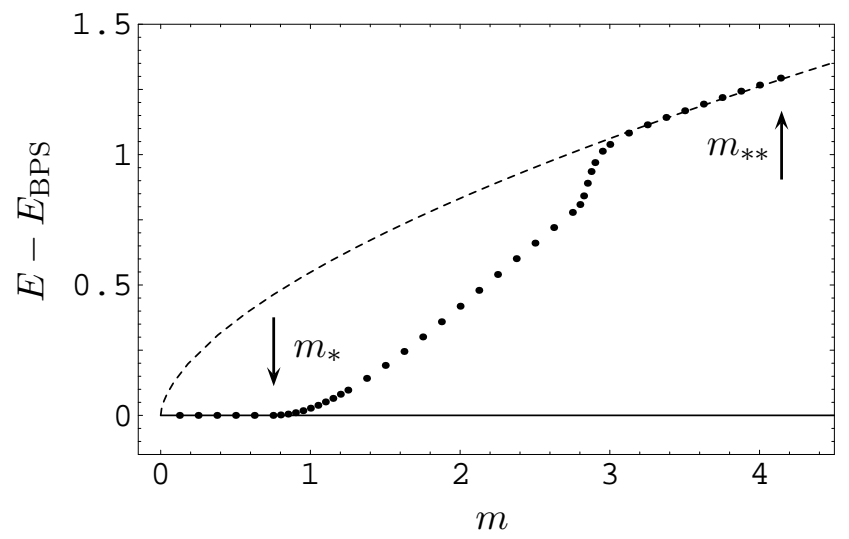

Figure 3: The difference between the energy $E$ of the domain wall configuration and the BPS bound $E_{\mathrm{BPS}}$ as a function of the mass $m$ (dots) for the $(5,3)$ theory. The dashed line indicates the difference $E_{2 \mathrm{~W}}-E_{\mathrm{BPS}}$.

is increased.

\section{The case $1 / 2 \leq N_{f} / N_{c}<1$}

If $1 / 2 \leq N_{f} / N_{c}<1$ there exist two BPS domain walls in the small $m$ limit. The argument that the number of BPS walls can only change by a multiple of two allows for the possible existence of two critical masses, $m_{*}$ and $m_{* *}$, as found in Ref.[2] for the theories $\left(N_{c}, N_{c}-1\right)$ and $N_{c}=2,3$ and 4 . At the mass $m_{*}$, the two BPS walls combine to form one non-BPS wall, and at the mass $m_{* *}$ the non-BPS wall disappears. We perform a numerical simulation of all theories in the class $1 / 2 \leq N_{f} / N_{c}<1$ with $N_{c} \leq 8$, and find the same sequence in each theory. The theories we simulated provide a sample of values of $N_{f} / N_{c}$ that covers the whole allowed range of $1 / 2 \leq N_{f} / N_{c}<1$. We therefore conclude that the theories in this class, not just the ones with $N_{c} \leq 8$, behave qualitatively in the same way. Of course, the actual values of $m_{*}$ and $m_{* *}$ depend on $N_{f} / N_{c}$.

In Fig. 3 we show how the difference between the energy of the domain wall and the BPS bound depends on the mass $m$ in a representative case, the $(5,3)$ theory. We have indicated the masses $m_{*}$ and $m_{* *}$, and we also plot the sum of the energy of the two BPS walls that connect the two vacua for $k=0$ and $k=1$ through the intermediate vacuum at $\phi=0$ and $\chi=0$,

$$
E_{2 \mathrm{~W}}=2 N_{c}\left(\frac{4}{3}\right)^{1-N_{f} / N_{c}} m^{N_{f} / N_{c}} .
$$

Below $m_{*}$, the energy of each of the two possible walls is equal to the BPS bound. When the mass is increased above $m_{*}$, the energy of the non-BPS wall starts to increase and tends towards $E_{2 \mathrm{~W}}$. In fact, at a mass below $m_{* *}$ the energy of the wall exceeds $E_{2 \mathrm{~W}}$. However, at that point there is still a sphaleron barrier, and the wall is meta-stable. The 


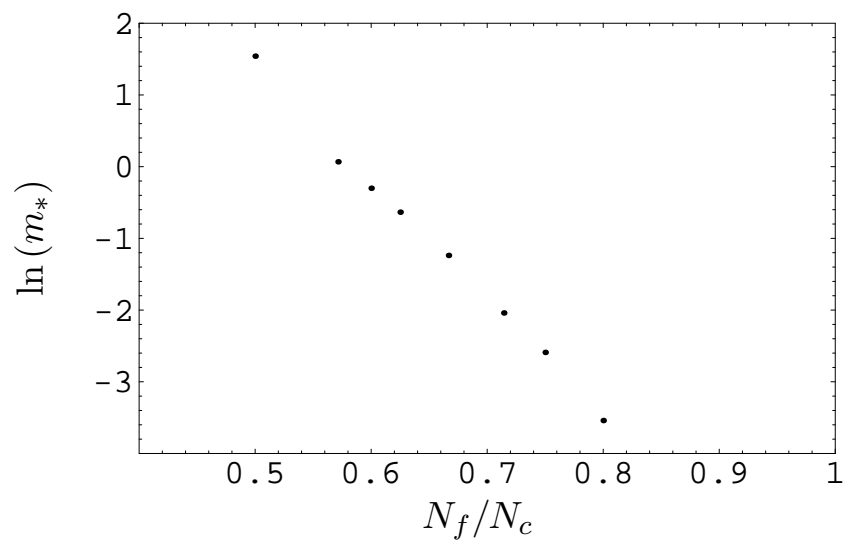

Figure 4: Logarithm of the critical mass $m_{*}$ versus the ratio $N_{f} / N_{c}$.

height of the barrier decreases as the mass approaches $m_{* *}$, and when the mass is equal to $m_{* *}$, the non-BPS wall ceases to exist.

Let us pause briefly to account for the kink in between $m_{*}$ and $m_{* *}$ appearing in Fig. 3 . At this value of the mass parameter $m$ the wall changes rapidly from a shape resembling the BPS configuration, to a shape more similar to a two wall configuration approaching the vacuum in the middle.

In order to find $m_{*}$ numerically, we start with a small value of the mass parameter $m$ for which the energy of the domain wall configuration does not differ significantly from the BPS bound. The initial configuration determines which of the two possible walls we obtain; this does not matter. Then we gradually increase $m$ and determine $m_{*}$ as the value of $m$ for which the energy of the wall differs significantly from the BPS bound.

In Table 1 we present the numerically determined values of the critical mass $m_{*}$ for the $\left(N_{c}, N_{f}\right)$ theories with $N_{c} \leq 8$. We also plot the logarithm of $m_{*}$ as a function of the ratio $N_{f} / N_{c}$ in Fig. 4. We are not able to determine $m_{*}$ numerically for $N_{f} / N_{c}>4 / 5$ because its value becomes too small. However, from Fig. 7 it appears that this critical mass goes to zero in the limit $N_{f} / N_{c} \rightarrow 1$.

In order to determine the critical mass $m_{* *}$, we use the following procedure. For a fixed value of $m$ we calculate the minimum energy $E$ for field configurations interpolating between the given vacua subject to a constrained value of $R_{0}$. If $m$ is just above $m_{*}$, a plot of $E$ versus $R_{0}$ shows a minimum and a maximum, corresponding to a non-BPS domain wall and a sphaleron, respectively. As $m$ increases the minimum and the maximum move towards each other and become less pronounced. We determine $m=m_{* *}$ as the value of $m$ were the maximum and the minimum coincide. For higher values of $m$, the energy $E$ increases monotonously with $R_{0}$, which means that the non-BPS wall has disappeared and the minimum energy configuration is formed by two walls connecting through the chirally symmetric vacuum.

In Fig. 5 we illustrate this procedure for the $(5,4)$ theory. The numerically determined values of the critical mass $m_{* *}$ for various $\left(N_{c}, N_{f}\right)$ theories are presented in Table \&. In 

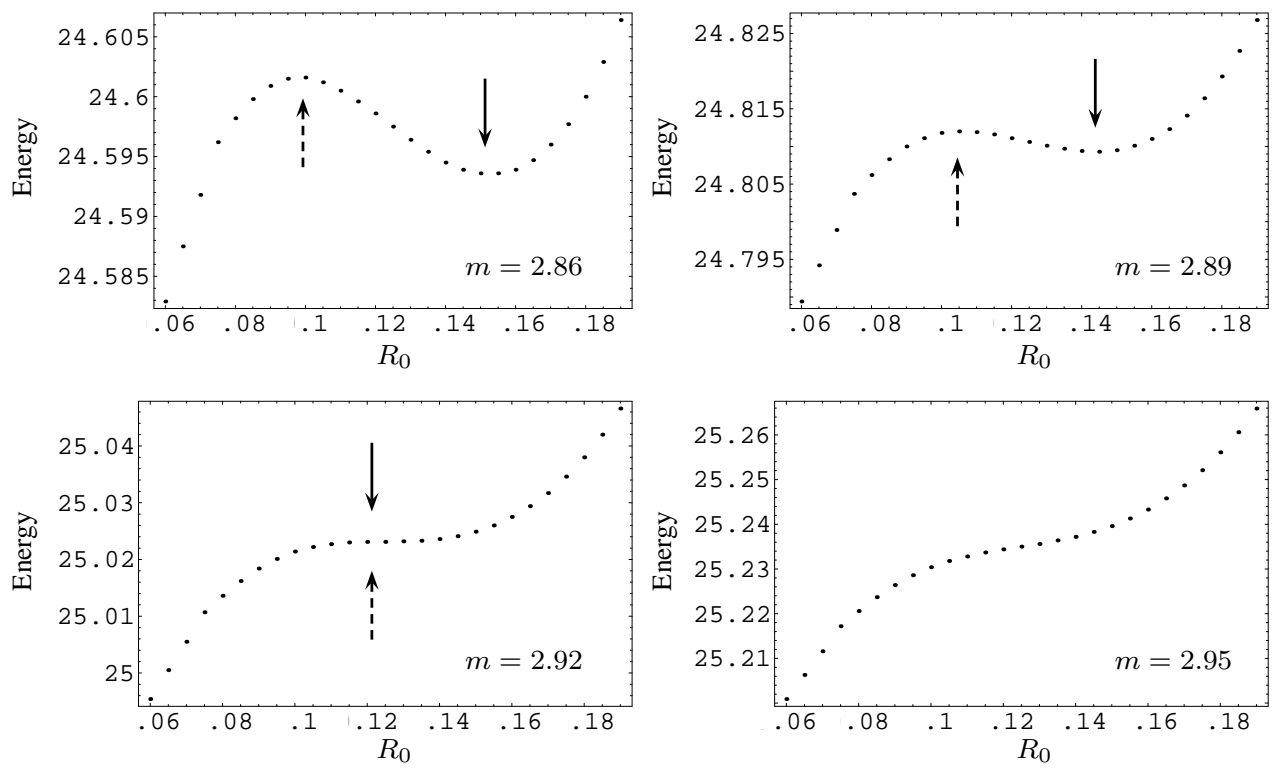

Figure 5: The minimum energy $E$ of interpolating field configurations subject to a constrained value of $R_{0}$ in the $(5,4)$ theory. The arrows indicate the position of the non-BPS domain wall (solid) and of the sphaleron (dashed). As $m$ increases, the minimum and the maximum move towards each other while the height of the barrier decreases. At $m_{* *}$ the wall and the sphaleron coincide and then they disappear.

Fig. 6 we plot $m_{* *}$ versus the ratio $N_{f} / N_{c}$. It can be seen from this figure that in the limit $N_{f} / N_{c} \rightarrow 1, m_{* *}$ tends to the constant value $m_{* *} \approx 1.83$.

\section{Discussion}

We derived our results in the context of the VYT effective Lagrangian. To what extend these results are valid for SQCD therefore depends on how well the VYT effective Lagrangian describes the vacuum structure of SQCD. An interesting discussion of this issue is given in Ref.[9].

In addition, we assumed that the Kähler potential for the fields $\Phi$ and $X$ has the canonical form (6). The tension of the BPS walls does not depend on the Kähler potential, but the shape of the domain walls and quantities such as the critical masses $m_{*}$ and $m_{* *}$ do depend on its details. It is instructive to see what happens in the case of a simple deformation of the Kähler potential,

$$
K=\alpha(\bar{\Phi} \Phi+\beta \bar{X} X)
$$

After rescaling $z^{\prime}=z / \alpha, X^{\prime}=\sqrt{\beta} X, m^{\prime}=m / \beta$ and $\Lambda^{\prime}=\Lambda \beta^{N_{f} /\left(3 N_{c}-N_{f}\right)}$, the static equation of motions of the scalar components are cast back into exactly the same form 


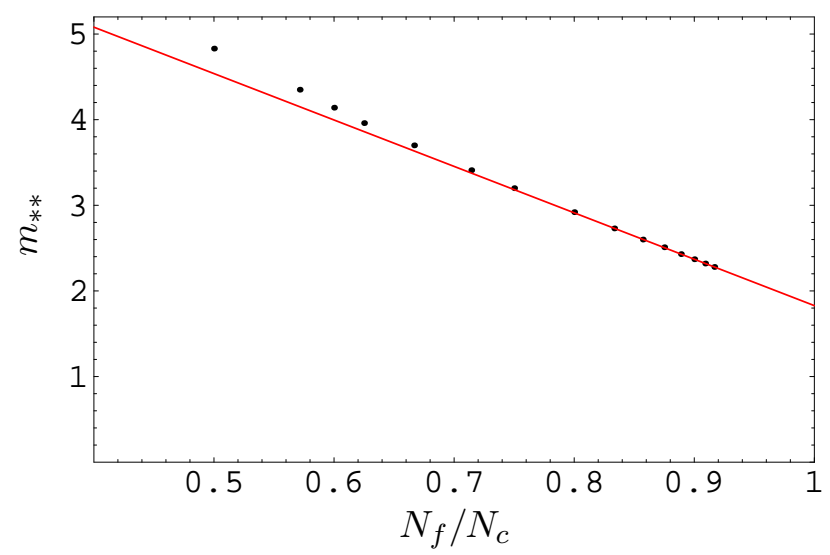

Figure 6: The critical mass $m_{* *}$ versus the ratio $N_{f} / N_{c}$. It can be seen that $m_{* *}$ approaches a constant value in the limit $N_{f} / N_{c} \rightarrow 1$. Extrapolating from our calculated points we find this limiting value to be $m_{* *} \approx 1.83$.

they have when $\alpha=\beta=1$, and our results apply to the primed quantities. Note that in principle both $\alpha$ and $\beta$ can depend on $N_{f}$ and $N_{c}$, and not necessarily only through the ratio $N_{f} / N_{c}$. Even when more complicated deformations of the Kähler potential are introduced, it is expected that the qualitative picture remains intact, at least as long as the singularity structure is not changed. In particular, for any value of $m$, the number of BPS walls will remain odd for $N_{f} / N_{c}<1 / 2$, and even for $1 / 2 \leq N_{f} / N_{c}<1$ under such deformations. Given the suspicion surrounding the chirally symmetric vacuum, it would be interesting to see what happens if our analysis were repeated with a Kähler potential that does not contain a singularity. The fate of the lower branch for theories with $N_{f} / N_{c}>1 / 2$ is uncertain in that case [10, 11], and our conclusions could potentially be changed.

Finally, the difference in the domain wall spectrum of the theories with $N_{f} / N_{c}<1 / 2$ and the theories with $1 / 2 \leq N_{f} / N_{c}<1$ poses the following question. What happens when the mass of one of the quark flavors in a theory with $N_{c}=3$ and $N_{f}=2$ is moved to infinity? The theory with two degenerate masses has an even number of BPS walls. If the mass of one of the quarks is taken to infinity while the other mass is kept finite, the heavy quark can be integrated out and the theory with $N_{c}=3$ and $N_{f}=1$ is obtained. However, this latter theory has only one BPS wall. This change in the number of BPS walls seems to be inconsistent with the argument that the number of BPS walls can only change by a multiple of two when a mass parameter is varied continuously. Obviously, one of the assumptions of the argument is violated. We intend to come back to this issue in a future work. 


\section{Acknowledgements}

The authors would like to thank A. Smilga, A. Vainshtein, B. de Carlos and V. Vento for interesting discussions. T.t.V. would like to thank the Departamento de Física Teórica of the Universidad de Valencia and the Instituto de Física Teórica in Sao Paulo, where part of this work was done, for warm hospitality. D.B. would like to thank the Theoretical Physics Institute of the University of Minnesota, where the final part of this work was done, and especially Professor Mikhail Shifman, for warm hospitality and partial support during his visit. The research of T.t.V. is supported in part by the Department of Energy under Grant No. DE-FG-94ER40823. The work of D.B. is supported by Ministerio de Educación y Cultura under Grant No. DGICYT-PB97-1227.

\section{References}

[1] G. Veneziano and S. Yankielowicz, Phys. Lett. B 113 (1982) 231; T. R. Taylor, G. Veneziano and S. Yankielowicz, Nucl. Phys. B 218 (1983) 493.

[2] A. Smilga and A. Veselov, Phys. Rev. Lett. 79 (1997) 4529 [hep-th/9706217; A. V. Smilga and A. I. Veselov, Nucl. Phys. B 515 (1998) 163 [hep-th/9710123; A. V. Smilga, Phys. Rev. D 58 (1998) 065005 [hep-th/9711032]; A. V. Smilga and A. I. Veselov, Phys. Lett. B 428 (1998) 303 [hep-th/9801142]; A. V. Smilga, in Continuous Advances in QCD 1998, World Scientific (1998) [hep-th/9807203.

[3] B. de Carlos and J. M. Moreno, Phys. Rev. Lett. 83 (1999) 2120 [hep-th/9905165]; B. de Carlos and J. M. Moreno, hep-th/9910208.

[4] A. Ritz, M. Shifman, A. Vainshtein and M. Voloshin, hep-th/0006028,

[5] M. Shifman, in Prog. Part. Nucl. Phys. 39 (1997) 1 [hep-th/9704114].

[6] A. Kovner and M. Shifman, Phys. Rev. D 56 (1997) 2396 [hep-th/9702174].

[7] A. Ritz and A. Vainshtein, Nucl. Phys. B566 (2000) 311 [hep-th/9909073].

[8] C. Csáki and H. Murayama, Nucl. Phys. B 515 (1998) 114.

[9] I. I. Kogan, A. Kovner and M. Shifman, Phys. Rev. D 57 (1998) 5195 [hepth/9712046].

[10] V. S. Kaplunovsky, J. Sonnenschein and S. Yankielowicz, Nucl. Phys. B552 (1999) 209 [hep-th/9811195].

[11] Y. Artstein, V. S. Kaplunovsky and J. Sonnenschein, hep-th/0010241.

[12] A. Gorsky, A. Vainshtein and A. Yung, Nucl. Phys. B584 (2000) 197 [hepth/0004087]. 
[13] B. Chibisov and M. Shifman, Phys. Rev. D 56 (1997) 7990 [hep-th/9706141]; G. W. Gibbons and P. K. Townsend, Phys. Rev. Lett. 83 (1999) 1727 [hepth/9905196].

[14] A. Kovner, M. Shifman and A. Smilga, Phys. Rev. D 56 (1997) 7978 [hepth/9706089]. 


\begin{tabular}{c||c|c|c|c|c|c|c|}
$\mathbf{N}_{\mathbf{f}} \backslash \mathbf{N}_{\mathbf{c}}$ & 2 & 3 & 4 & 5 & 6 & 7 & 8 \\
\hline \hline 1 & 4.67 & $\mathrm{x}$ & $\mathrm{x}$ & $\mathrm{x}$ & $\mathrm{x}$ & $\mathrm{x}$ & $\mathrm{x}$ \\
\hline 2 & & 0.29 & $(2,1)$ & $\mathrm{x}$ & $\mathrm{x}$ & $\mathrm{x}$ & $\mathrm{x}$ \\
\hline 3 & & & 0.075 & 0.74 & $(2,1)$ & $\mathrm{x}$ & $\mathrm{x}$ \\
\hline 4 & & & & 0.029 & $(3,2)$ & 1.07 & $(2,1)$ \\
\hline 5 & & & & & $?$ & 0.13 & 0.47 \\
\hline 6 & & & & & & $?$ & $(4,3)$ \\
\hline 7 & & & & & & & $?$ \\
\hline
\end{tabular}

Table 1: Numerically determined values of the critical mass $m_{*}$ for different $\left(N_{c}, N_{f}\right)$ theories. The symbol ' $\mathrm{x}$ ' indicates that the corresponding theory has one BPS wall for any value of the mass parameter $m$ and therefore there is no $m_{*}$. The question mark indicates that the value of $m_{*}$ is too small to be determined by our method.

\begin{tabular}{c|c|c|c|c|c|c|c|}
$\mathbf{N}_{\mathbf{f}} \mathbf{N}_{\mathbf{c}}$ & 2 & 3 & 4 & 5 & 6 & 7 & 8 \\
\hline \hline 1 & 4.83 & $\mathrm{x}$ & $\mathrm{x}$ & $\mathrm{x}$ & $\mathrm{x}$ & $\mathrm{x}$ & $\mathrm{x}$ \\
\hline 2 & & 3.70 & $(2,1)$ & $\mathrm{x}$ & $\mathrm{x}$ & $\mathrm{x}$ & $\mathrm{x}$ \\
\hline 3 & & & 3.20 & 4.14 & $(2,1)$ & $\mathrm{x}$ & $\mathrm{x}$ \\
\hline 4 & & & & 2.92 & $(3,2)$ & 4.35 & $(2,1)$ \\
\hline 5 & & & & & 2.73 & 3.41 & 3.96 \\
\hline 6 & & & & & & 2.60 & $(4,3)$ \\
\hline 7 & & & & & & & 2.51 \\
\hline
\end{tabular}

Table 2: Numerically determined values of the critical mass $m_{* *}$. The symbol ' $x$ ' indicates that the corresponding theory has one BPS wall for any value of the mass parameter $m$ and therefore there is no $m_{* *}$. 